\title{
A Transgenic Mouse Model for Studying the Role of the Parathyroid Hormone-Related Protein System in Renal Injury
}

\author{
Ricardo J. Bosch, ${ }^{1}$ Arantxa Ortega, ${ }^{1}$ Adriana Izquierdo, ${ }^{1}$ Ignacio Arribas, ${ }^{2}$ \\ Jordi Bover, ${ }^{3}$ and Pedro Esbrit ${ }^{4}$ \\ ${ }^{1}$ Laboratory of Renal Physiology and Experimental Nephrology, Department of Physiology, School of Medicine, Alcalá University, \\ University Campus, 28871 Alcalá de Henares, Spain \\ ${ }^{2}$ Department of Biochemistry, 'Principe de Asturias' University Hospital, University of Alcalá, 28871 Alcalá de Henares, Spain \\ ${ }^{3}$ Nephrology Department, Puigvert Foundation, 08025 Barcelona, Spain \\ ${ }^{4}$ Bone and Mineral Metabolism Laboratory, Biomedical Research Institute-Jiménez Díaz, 28040 Madrid, Spain
}

Correspondence should be addressed to Ricardo J. Bosch, ricardoj.bosch@uah.es

Received 13 September 2010; Accepted 11 October 2010

Academic Editor: Oreste Gualillo

Copyright () 2011 Ricardo J. Bosch et al. This is an open access article distributed under the Creative Commons Attribution License, which permits unrestricted use, distribution, and reproduction in any medium, provided the original work is properly cited.

\begin{abstract}
Parathyroid hormone- (PTH-) related protein (PTHrP) and its receptor, the PTH1 receptor (PTH1R), are widely expressed in the kidney, where PTHrP exerts a modulatory action on renal function. PTHrP is known to be upregulated in several experimental nephropathies such as acute renal failure (ARF), obstructive nephropathy (ON) as well as diabetic nephropathy (DN). In this paper, we will discuss the functional consequences of chronic PTHrP overexpression in the damaged kidney using a transgenic mouse strain overexpressing PTHrP in the renal proximal tubule. In both ARF and ON, PTHrP displays proinflammatory and profibrogenic actions including the induction of epithelia to mesenquima transition. Moreover, PTHrP participates in the mechanisms of renal hypertrophy as well as proteinuria in experimental DN. Angiotensin II (Ang II), a critical factor in the progression of renal injury, appears to be, at least in part, responsible for endogenous PTHrP upregulation in these pathophysiological settings. These findings provide novel insights into the well-known protective effects of Ang II antagonists in renal diseases, paving the way for new therapeutic approaches.
\end{abstract}

\section{Introduction}

Parathyroid hormone- (PTH-)related protein (PTHrP) was discovered at the end of the 1980 s as the factor responsible for humoral hypercalcemia of malignancy [1]. However, while PTH is a well-characterized endocrine regulator of mineral homeostasis, PTHrP is widely expressed in nonmalignant fetal and adult tissues [2]. Despite the widespread production of PTHrP in normal tissues, its circulating concentration in healthy subjects is below the detectable limit in the majority of current assays [3]. Thus, in contrast to the situation of humoral hypercalcemia of malignancy in which PTHrP plays the role of a classic hormone, this protein exerts paracrine, autocrine, and/or intracrine actions in normal tissues. PTHrP is a key regulator of placental calcium transport in the foetus, and it appears to be a physiological modulator of smooth muscle tone. Current concepts indicate that PTHrP is a developmental and/or growth-regulating factor, much more similar to other known cytokines and growth factors than to PTH $[1,2]$.

In the adult kidney, both parathyroid hormone-(PTH-) related protein (PTHrP) and the PTH1 receptor (PTH1R) are abundant throughout the renal parenchyma, including the intrarenal vasculature [4-6]. In the kidney, PTHrP appears to modulate renal plasma flow and glomerular filtration rate, and induces proliferative effects on both glomerular mesangial and tubuloepithelial cells [7-14].

Pioneer studies from Soifer et al. [11] at the beginning of the 1990's suggested the implication of PTHrP in the mechanisms of injury and/or repair of the tubular epithelium 
after acute renal failure (ARF). Proliferation of injured tubule cells appears to be important for timely tubular recovery after renal injury, and for subsequent functional recovery of the damaged kidney. Several growth factors and cytokines, acting in an autocrine and paracrine manner, appear to participate in the repair process of the tubular epithelium in this setting [15-19]. The mitogenic features of PTHrP and its early overexpression after renal injury in experimental models of ARF induced by either ischemia or nephrotoxins initially suggested that PTHrP could participate in the regenerative process after ARF $[11,13]$. The recent development of a transgenic mouse model characterized by PTHrP overexpression in the renal proximal tubule made it possible to explore the functional consequences of chronic PTHrP overexpression in both glomerular and tubular experimental models of renal damage. This novel approach has provided valuable data which have helped to disclose the true roles of PTHrP in the damaged kidney. The following paragraphs describe the results of over a decade of intense investigation on this issue.

\section{The PTHrP-Overexpressing Mice}

A transgenic mouse strain characterized by PTHrP overexpression in the renal proximal tubule was developed at Yale University a few years ago [15]. The renal specificity of the transgene was conferred by the $\gamma$-glutamyl transpeptidase1 (GGT-1) promoter, mainly expressed in the renal proximal tubule. PTHrP-overexpressing mice were generated by breeding two types of transgenic mice: one containing a GGT-1 promoter fragment upstream of a tetracycline transactivator fusion protein, which functions as a strong transcription activator, and the other with a PTHrP cDNA placed under the control of a tetracycline operator construct (Figure 1). The tetracycline-controlled transactivator strategy, which creates a reversible switch "on/off" for gene expression, ensures the temporal control of PTHrP gene activity. Transgene-bearing founders were continuously outbred to normal CD-1 mice to generate hemizygotes. Transgenic founders were identified by PCR analysis of tail DNA using specific primers [15]. Overexpression of PTHrP, in the renal proximal tubule, did not alter the normal level of expression of endogenous PTHrP. Renal PTHrP protein levels in PTHrP-overexpressing animals were 3-4 times over those in their control littermates, as confirmed by Western blot; but its circulating levels were undetectable $(<0.2 \mathrm{pM})$ in these animals [15]. The renal protein levels of the PTH1R, analyzed by Western blot, were similar to those in control mice, indicating that this receptor expression is not influenced by PTHrP upregulation in these mice [15]. Analysis of kidney size and morphology, and serum creatinine levels revealed no significant differences between PTHrP-overexpressing mice and their control littermates, indicating a normal growth and basal function of the mouse kidney in the former mice [15]. The functional consequences of chronic PTHrP overexpression have been extensively studied in recent years using this transgenic mouse model and experimental nephropathies with predominant affection at tubular and glomerular level.

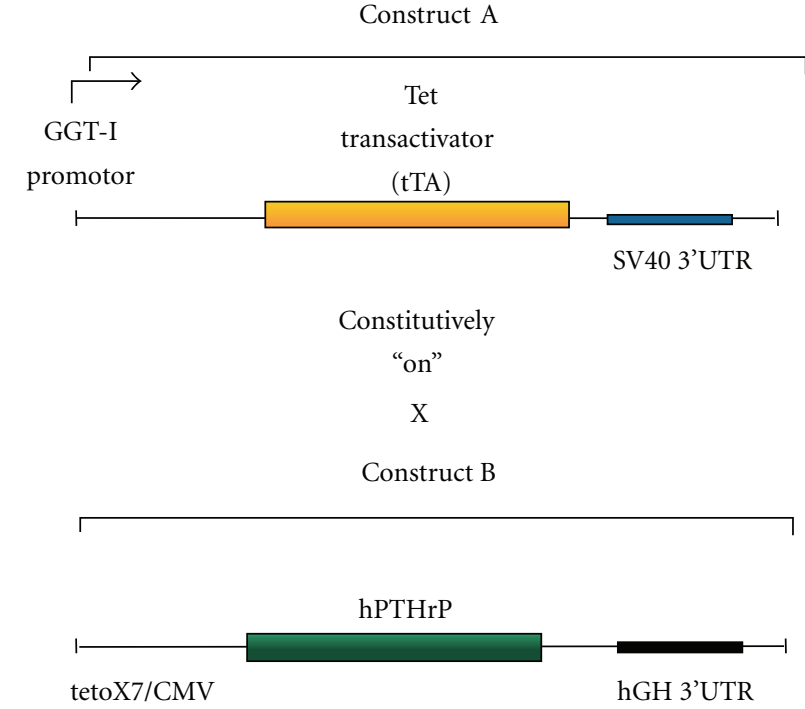

Figure 1: Schematic representation of the different transgenes used to generate the PTHrP-overexperssing mice. Construct A, containing sequences for the $\gamma$-glutamyl transpeptidase-I (GGTI) promoter, cDNA sequences for the Tet transactivator protein (tTA), and the SV-40 T antigen $3^{\prime}$ UTR, was used to generate GGT-tTA transgenic mice, resulting in specific expression of the tTA protein in the renal proximal tubule cells. Construct B, containing sequences for a hybrid regulatory element composed of a heptamerized tetracycline operator (TetoX7) fused to a minimal human cytomegalovirus promoter element, the human growth hormone (hGH) 3' UTR, and hPTHrP (1-141) cDNA sequences, was used to generate Teto-PTHrP transgenic mice. Hemizygote mice bearing the construct A were bred with construct B-bearing hemizygote mice to induce PTHrP overexpression in renal proximal tubule cells.

2.1. PTHrP in Renal Inflammation. Tubulointerstitial inflammation is a key event in a variety of nephropathies. Early after renal injury, damaged tubuloepithelial cells begin to overexpress proinflammatory cytokines and chemokines, which promote migration of monocytes/macrophages and T-lymphocytes to the renal interstitium [20, 21]. Both infiltrating leukocytes and damaged tubuloepithelial cells activate and induce proliferation of resident fibroblasts in the tubulointerstitial compartment. A severe and prolonged injury will determine a sustained activation of proinflammatory pathways, associated with overexpression of profibrogenic cytokines by tubulointerstitial cells leading to fibrogenesis and renal function loss [22].

Early studies suggested that PTHrP might act as an important mediator of proinflammatory cytokines, namely, tumour necrosis factor and interleukin-6, in multiorgan inflammation and rheumatoid arthritis [23]. More recent studies have shown that PTHrP activates nuclear factor $(\mathrm{NF})-\kappa \mathrm{B}$ and the expression of NF- $\kappa \mathrm{B}$-dependent cytokines and chemokines [e.g., IL-6 and monocyte chemoattractant protein-1 (MCP-1) in different cell types [24, 25]. PTHrP and MCP-1 were found to colocalize in smooth muscle cells in human atherosclerotic plaques [25-27]. 
Injury

(nephrotoxic, ischemia, hyperglycemia)
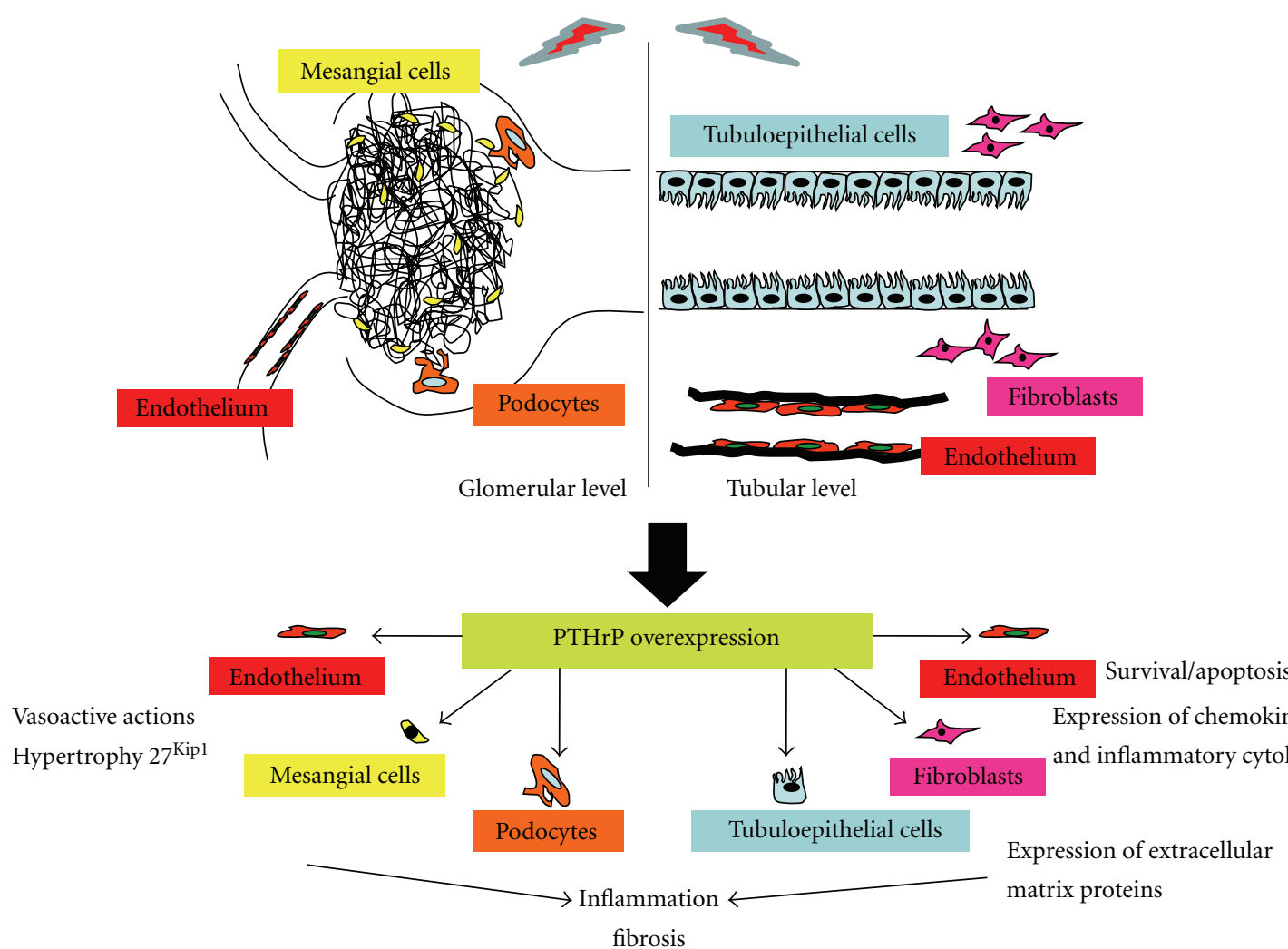

FIGURE 2: Schematic representation of the different actions whereby PTHrP might promote inflammation and fibrogenesis in the injured kidney.

Moreover, in mice with unilateral ureteral obstruction (UUO), a well-characterized model of renal inflammation we recently showed that PTHrP was upregulated in the obstructed kidney, even in PTHrP-overexpressing mice [28]. In contrast to previous observations in ischemic or nephrotoxic renal injury $[8,11,13]$, PTH1R was not downregulated after UUO in mice [28]. Interestingly, upregulation of both PTHrP and PTH1R was also recently observed in the kidney of diabetic mice [29]. Furthermore, our recent in vitro findings indicate that $\mathrm{PTHrP}$ augments the production of several proinflammatory factors in tubuloepithelial cell and promotes monocyte/macrophage migration [28]. Extracellular signal-regulated kinase-(ERK-) mediated NF$\kappa \mathrm{B}$ activation appears to be an important mechanism whereby PTHrP triggers renal inflammation [28]. Therefore, PTHrP might be envisioned as a new inflammation marker and a potential therapeutic target in the obstructed kidney. Finally, since sustained renal inflammation is closely related to fibrogenesis, these data point to PTHrP as a likely proinflammatory and profibrogenic cytokine in the damaged kidney.

2.2. PTHrP in Renal Cell Apoptosis. Apoptosis is considered to be an important component of the acute response of the tubular epithelium to injury [30, 31]. Our data, using PTHrP-overexpressing mice with folic acid-induced ARF, suggest that this PTHrP action might have detrimental consequences in the injured kidney. These mice showed a significant delay in renal function recovery and higher focal areas of tubulointerstitial fibrosis than normal mice, associated with a decrease in apoptotic tubulointerstitial cells [32]. The rationale for this association might come from the fact that apoptosis of interstitial fibroblasts appears to be a mechanism to prevent fibrogenesis [16-22].

2.3. PTHrP in Diabetic Nephropathy. Recently, we hypothesized that PTHrP involvement in the mechanisms of renal injury might not be limited to conditions with predominant damage of the renal tubulointerstitium, and might be extended to glomerular diseases, such as DN. Thus, using an experimental model of DN induced by streptozotocin (STZ) [29], we studied the possible changes in the PTHrP/PTH1R system associated with the outcome of this nephropathy, characterized by an initial phase of renal hypertrophy at both tubular and glomerular levels, followed by an increase in urinary albumin excretion (UAE) (proteinuria) $[33,34]$. DN was induced in Swiss-CD1 (CD1) mice as well as in PTHrPoverexpressing mice. In the diabetic $\mathrm{CD}-1$ mice, a significant 
increase in the expression of both PTHrP and PTH1R was observed, at both glomerular and tubular levels, associated with the development of an increase in the UAE [29]. On the other hand, diabetic PTHrP-overexpressing mice, in comparison to their control littermates, have increased renal hypertrophy, a significant higher UAE and lower total plasma protein levels. A significant association among the renal expression of PTHrP, PTH1R, and UAE was found to occur in the diabetic mice. Furthermore, there was a 6 -fold increase in the risk of developing proteinuria in those mice with the higher PTHrP and PTH1R levels, according to the logistic regression analysis [29]. It is interesting to mention that, albeit the STZ model has limitations for assessing longterm histomorphological changes in the diabetic kidney [33], the aforementioned findings might have pathophysiological implications since the amount of proteinuria is a reliable predictor of diabetic nephropathy [34].

More recently, the putative role of PTHrP in the hypertrophy of the diabetic kidney was explored. It is well established that high glucose (HG) leading to renal cell hypertrophy appears to involve cell entry into the cell cycle and subsequent arrest at the G1/S interphase, followed by an increase in cell protein synthesis. Recent studies have shown that HG-induced glomerular cell hypertrophy (affecting both mesangial cells and podocytes) involves an early activation of the renin-angiotensin system, followed by an induction of TGF- $\beta_{1}$, which in turn activates a cell cycle regulatory protein, the cycline-dependent kinase inhibitor (CDKI) p27 Kip1 [35-37]. In mouse podocytes grown in HG condition, Romero et al. found that Ang II induces PTHrP upregulation, which in turn triggers both TGF- $\beta_{1}$ and $\mathrm{p} 27^{\mathrm{Kip} 1}$ overexpression, and thereby promotes podocyte hypertrophy [38]. Interestingly, the latter factors were found to be constitutively upregulated in nondiabetic PTHrPoverexpressing mice [38].

Collectively, these data indicate that the renal PTHrP/ PTH1R system is upregulated in STZ-induced diabetic mice, where it appears to be involved in renal hypertrophy and adversely affects the outcome of DN. Furthermore, current findings also suggest that PTHrP can participate in the hypertrophy mechanism(s) targeted by HG in podocytes.

2.4. PTHrP in Renal Fibrosis. Renal fibrosis is recognized as the final common stage of main renal diseases, capable of progressing to chronic renal failure. Interstitial fibroblasts are the main cell type responsible for fibrogenesis, a process in which these cells proliferate and become activated myofibroblasts [39]. Fibrosis of the kidney is known to be induced by both tubuloepithelial and infiltrating cells, as well as secretion of matrix compounds by both activated fibroblasts and tubular cells. In fact, an increased matrix synthesis and deposition, and loss of tubular structural integrity, are paramount events at later stages of fibrogenesis [40]. Previous studies indicate that the higher number of infiltrating macrophages was associated with increased fibroblast proliferation in the renal interstitium of folic acid-injured kidneys from PTHrP-overexpressing mice [32]. In these mice, an increased immunostaining for $\alpha$-smooth muscle actin (SMA), a marker of activated fibroblasts or myofibroblasts [41], was also observed in the renal interstitium after folic acid nephrotoxicity [32]. Consistent with the latter in vivo findings, PTHrP $[1-35,42]$ was found to stimulate $\alpha$-SMA expression in renal fibroblasts in vitro [32]. In addition, a higher immunostaining for both types I and IV collagens was observed in the renal interstitium of the obstructed kidneys from PTHrP-overexpressing mice, than in their normal littermates [43]. In agreement with these in vivo findings, PTHrP [1-36] was found to stimulate the expression of type- 1 procollagen and fibronectin in tubuloepithelial cells and renal fibroblasts in vitro. At least part of these effects was abolished by a PTH1R antagonist [32].

Tubuloepithelial cells might also contribute to the development of renal fibrosis by directly generating myofibroblasts through a process known as epithelial-mesenchymal transition (EMT) $[43,44]$. EMT is a multiple step process that requires the integration of several extrinsic and intrinsic pathways including loss of epithelia polarity and rearrangement of the F-actin cytoskeleton, associated with upregulation of genes used as EMT markers [45]. The latter includes, in addition to $\alpha$-SMA which increases cell contractility and motility, extracellular matrix proteins such as fibronectin and several types of collagens, metalloproteases, and integrinlinked kinase. In addition, a decrease in the expression of proteins that keep basolateral polarity and intracellular junctions, including cytokeratin and the adherent junction proteins E-cadherin and $\beta$-catenin, takes place in the renal tubuloepithelium during EMT [41, 45-49].

Recently, Ardura et al. [39, 43] expanded these studies and showed that PTHrP is capable of inducing a variety of phenotypic changes related to EMT in tubuloepithelial cells. Moreover, PTHrP can upregulate both TGF- $\beta_{1}$ and vascular endothelial growth factor (VEGF) expression in these cells. Also, blockade of these growth factors by different manoeuvres was found to diminish both EMT changes in cultured renal epithelial cells. Of note in this regard, a VEGF antibody also decreased the renal fibrosis in the obstructed mouse kidney [43]. Hence, both VEGF and TGF- $\beta_{1}$ are likely to act as downstream mediators of PTHrP deleterious actions in the damaged kidney. Interestingly, a similar interaction between the two latter factors was recently observed in the setting of PTHrP-induced podocyte hypertrophy. Recent evidences strongly suggest that PTHrP, TGF- $\beta$, VEGF, as well as activation of the epidermal growth factor receptor (EGFR), might all act in concert through activation of ERK to induce EMT in renal tubuloepithelial cells [39].

EMT-related changes have been found to occur in the mouse obstructed kidney associated with constitutive PTHrP overexpression [39]. Two important EMT mediators, namely, TGF- $\beta_{1}$ and $\mathrm{p}$-EGFR proteins were upregulated in the obstructed kidney of these transgenic mice, suggesting that PTHrP might also interact with the aforementioned factors to modulate EMT in vivo [39].

Collectively, all the available data demonstrate a major role for PTHrP in renal fibrogenesis, due to its capacity to induce the expression of extracellular matrix proteins as well as by modulating EMT in renal tubuloepithelial cells. 


\section{Interaction between PTHrP and Angiotensin II in the Damaged Kidney}

The renin-angiotensin system is well known for playing an important pathogenic role in the mechanisms of renal injury $[50,51]$. Local activation of components of this system, including Ang II, in the kidney has shown to occur early in various experimental models of ARF, for example, folic acidinduced nephrotoxicity and ischemia/reperfusion $[13,52-$ 54]. Moreover, Ang II antagonists exert beneficial effects on renal function in these models $[52,55,56]$.

Recent data strongly suggest that PTHrP might be involved in the mechanisms related to Ang II-induced renal injury. Exogenously administered Ang II, via its type 1 (AT1) receptor, increases PTHrP expression in glomerular and tubular cells as well as in vascular smooth muscle cells both in vivo and in vitro [57-59]. Interestingly, a significant correlation between PTHrP overexpression and tubular damage and fibrosis was observed in the rat kidney after systemic Ang II infusion [57]. Furthermore, in nephrotoxic ARF, the improvement of renal function by Ang II antagonists was associated with inhibition of PTHrP overexpression [52]. These aggregated data suggest that Ang II is a likely candidate responsible for PTHrP overexpression, and this might contribute to the deleterious effects of Ang II in the damaged kidney. These findings could provide novel insights into the well-known protective effects of Ang II antagonists in renal diseases, possibly leading the way to new therapeutic approaches.

\section{Conclusion}

The upregulation of the renal PTHrP system, which occurs at least in part through Ang II, represents a common event in several experimental nephropathies, namely, ARF and DN. In the former condition, PTHrP appears to contribute to the progression of renal damage by increasing tubulointerstitial cell survival, inflammation, and fibrogenesis in part through promoting EMT. In DN, PTHrP can promote renal hypertrophy and proteinuria (Figure 2). Collectively, both in vitro and in vivo findings in transgenic $\mathrm{PTHrP}$-overexpressing mice strongly support the role of PTHrP as a novel pathogenic factor in kidney disease, and also provide novel insights into the protective effects of Ang II antagonists in various nephropathies.

\section{Acknowledgments}

A. Ortega is the recipient of a research contract from the Comunidad Autónoma de Madrid (S-BIO-2083-2006). A. Izquierdo is currently Assistant Professor at the Rey Juan Carlos University, Alcorcón, Madrid. The studies included in this paper were supported in part by Grants from Ministerio de Educación y Cultura of Spain (SAF2002-04356-C03-01, -02, and -03), Ministerio de Ciencia e Innovación (SAF200912009-C02-01), Comunidad Autónoma de Madrid (CAM 08.6/0038.1/2000-2 and GR/SAL/0415/2004), Instituto de Salud Carlos III (RETICEF RD06/0013/1002), Spanish
Society of Nephrology, Eugenio Rodríguez Pascual Foundation, and Conchita Rábago de Jiménez Díaz Foundation.

\section{References}

[1] T. J. Martin, J. M. Moseley, and E. D. Williams, "Parathyroid hormone-related protein: hormone and cytokine," Journal of Endocrinology, vol. 154, supplement, pp. S23-S37, 1997.

[2] W. M. Philbrick, J. J. Wysolmerski, S. Galbraith et al., "Defining the roles of parathyroid hormone-related protein in normal physiology," Physiological Reviews, vol. 76, no. 1, pp. 127-173, 1996.

[3] F. de Miguel, J. L. Motellón, J. Hurtado, F. J. Jiménez, and P. Esbrit, "Comparison of two immunoradiometric assays for parathyroid hormone-related protein in the evaluation of cancer patients with and without hypercalcemia," Clinica Chimica Acta, vol. 277, no. 2, pp. 171-180, 1998.

[4] T. L. Clemens, S. Cormier, A. Eichinger et al., "Parathyroid hormone-related protein and its receptors: nuclear functions and roles in the renal and cardiovascular systems, the placental trophoblasts and the pancreatic islets," British Journal of Pharmacology, vol. 134, no. 6, pp. 1113-1136, 2001.

[5] T. Massfelder, A. F. Stewart, K. Endlich, N. Soifer, C. Judes, and J.-J. Helwig, "Parathyroid hormone-related protein detection and interaction with NO and cyclic AMP in the renovascular system," Kidney International, vol. 50, no. 5, pp. 1591-1603, 1996.

[6] T. Yang, S. Hassan, Y. G. Huang, A. M. Smart, J. P. Briggs, and J. B. Schnermann, "Expression of PTHrP, PTH/PTHrP receptor, and $\mathrm{Ca}^{2+}$-sensing receptor mRNAS along the rat nephron," American Journal of Physiology, vol. 272, no. 6, pp. F751-F758, 1997.

[7] R. J. Bosch, P. Rojo-Linares, G. Torrecillas-Casamayor, M. C. Iglesias-Cruz, D. Rodríguez-Puyol, and M. Rodríguez-Puyol, "Effects of parathyroid hormone-related protein on human mesangial cells in culture," American Journal of Physiology, vol. 277, no. 6, pp. E990-E995, 1999.

[8] P. Esbrit, S. Santos, A. Ortega et al., "Parathyroid hormonerelated protein as a renal regulating factor: from vessels to glomeruli and tubular epithelium," American Journal of Nephrology, vol. 21, no. 3, pp. 179-184, 2001.

[9] T. Massfelder, N. Parekh, K. Endlich, C. Saussine, M. Steinhausen, and J.-J. Helwig, "Effect of intrarenally infused parathyroid hormone-related protein on renal blood flow and glomerular filtration rate in the anaesthetized rat," British Journal of Pharmacology, vol. 118, no. 8, pp. 1995-2000, 1996.

[10] N. Endlich, R. Nobiling, W. Kriz, and K. Endlich, "Expression and signaling of parathyroid hormone-related protein in cultured podocytes," Experimental Nephrology, vol. 9, no. 6, pp. 436-443, 2001.

[11] N. E. Soifer, S. K. Van Why, M. B. Ganz, M. Kashgarian, N. J. Siegel, and A. F. Stewart, "Expression of parathyroid hormonerelated protein in the rat glomerulus and tubule during recovery from renal ischemia," Journal of Clinical Investigation, vol. 92, no. 6, pp. 2850-2857, 1993.

[12] A. García-Ocaña, F. de Miguel, C. Peñaranda, J. P. Albar, J. L. Sarasa, and P. Esbrit, "Parathyroid hormone-related protein is an autocrine modulator of rabbit proximal tubule cell growth," Journal of Bone and Mineral Research, vol. 10, no. 12, pp. 1875-1884, 1995.

[13] S. Santos, R. J. Bosch, A. Ortega et al., "Up-regulation of parathyroid hormone-related protein in folic acid-induced acute renal failure," Kidney International, vol. 60, no. 3, pp. 982-995, 2001. 
[14] R. Largo, D. Gómez-Garre, S. Santos et al., "Renal expression of parathyroid hormone-related protein (PTHrP) and $\mathrm{PTH} / \mathrm{PTHrP}$ receptor in a rat model of tubulointerstitial damage," Kidney International, vol. 55, no. 1, pp. 82-90, 1999.

[15] N. M. Fiaschi-Taesch, S. Santos, V. Reddy et al., "Prevention of acute ischemic renal failure by targeted delivery of growth factors to the proximal tubule in transgenic mice: the efficacy of parathyroid hormone-related protein and hepatocyte growth factor," Journal of the American Society of Nephrology, vol. 15, no. 1, pp. 112-125, 2004.

[16] H. D. Humes, E. W. Lake, and S. Liu, "Renal tubule cell repair following acute renal injury," Mineral and Electrolyte Metabolism, vol. 21, no. 4-5, pp. 353-365, 1995.

[17] S. Vukicevic, V. Basic, D. Rogic et al., "Osteogenic protein1 (bone morphogenetic protein-7) reduces severity of injury after ischemic acute renal failure in rat," Journal of Clinical Investigation, vol. 102, no. 1, pp. 202-214, 1998.

[18] K. Matsumoto and T. Nakamura, "Hepatocyte growth factor: renotropic role and potential therapeutics for renal diseases," Kidney International, vol. 59, no. 6, pp. 2023-2038, 2001.

[19] G. Gobé, X.-J. Zhang, D. A. Willgoss, E. Schock, N. A. Hogg, and Z. H. Endre, "Relationship between expression of Bcl-2 genes and growth factors in ischemic acute renal failure in the rat," Journal of the American Society of Nephrology, vol. 11, no. 3, pp. 454-467, 2000.

[20] A. J. Rees, "The role of infiltrating leukocytes in progressive renal disease: implications for therapy," Nature Clinical Practice Nephrology, vol. 2, no. 7, pp. 348-349, 2006.

[21] G. A. Müller, J. Markovic-Lipkovski, J. Frank, and H. P. Rodemann, "The role of interstitial cells in the progression of renal diseases," Journal of the American Society of Nephrology, vol. 2, no. 10, pp. S198-S205, 1992.

[22] F. Strutz and E. G. Neilson, "New insights into mechanisms of fibrosis in immune renal injury," Springer Seminars in Immunopathology, vol. 24, no. 4, pp. 459-476, 2003.

[23] J. L. Funk, "A role for parathyroid hormone-related protein in the pathogenesis of inflammatory/autoimmune diseases," International Immunopharmacology, vol. 1, no. 6, pp. 11011121, 2001.

[24] C. Guillén, P. Martínez, A. R. De Gortázar, M. E. Martínez, and P. Esbrit, "Both N- and C-terminal domains of parathyroid hormone-related protein increase interleukin- 6 by nuclear factor- $\kappa \mathrm{B}$ activation in osteoblastic cells," The Journal of Biological Chemistry, vol. 277, no. 31, pp. 28109-28117, 2002.

[25] J. L. Martín-Ventura, M. Ortego, P. Esbrit, M. A. HernándezPresa, L. Ortega, and J. Egido, "Possible role of parathyroid hormone-related protein as a proinflammatory cytokine in atherosclerosis," Stroke, vol. 34, no. 7, pp. 1783-1789, 2003.

[26] M. Ishikawa, M. Akishita, K. Kozaki et al., "Expression of parathyroid hormone-related protein in human and experimental atherosclerotic lesions: functional role in arterial intimal thickening," Atherosclerosis, vol. 152, no. 1, pp. 97-105, 2000.

[27] T. Nakayama, A. Ohtsuru, H. Enomoto et al., "Coronary atherosclerotic smooth muscle cells overexpress human parathyroid hormone-related peptides," Biochemical and Biophysical Research Communications, vol. 200, no. 2, pp. 10281035, 1994.

[28] D. Rámila, J. A. Ardura, V. Esteban et al., "Parathyroid hormone-related protein promotes inflammation in the kidney with an obstructed ureter," Kidney International, vol. 73, no. 7, pp. 835-847, 2008.

[29] A. Izquierdo, P. López-Luna, A. Ortega et al., "The parathyroid hormone-related protein system and diabetic nephropathy outcome in streptozotocin-induced diabetes," Kidney International, vol. 69, no. 12, pp. 2171-2178, 2006.

[30] G. P. Kaushal, A. G. Basnakian, and S. V. Shah, "Apoptotic pathways in ischemic acute renal failure," Kidney International, vol. 66, no. 2, pp. 500-506, 2004.

[31] A. G. Basnakian, G. P. Kaushal, and S. V. Shah, "Apoptotic pathways of oxidative damage to renal tubular epithelial cells," Antioxidants and Redox Signaling, vol. 4, no. 6, pp. 915-924, 2002.

[32] A. Ortega, D. Rámila, J. A. Ardura et al., "Role of parathyroid hormone-related protein in tubulointerstitial apoptosis and fibrosis after folic acid-induced nephrotoxicity," Journal of the American Society of Nephrology, vol. 17, no. 6, pp. 1594-1603, 2006.

[33] M. L. Gross, E. Ritz, A. Schoof et al., "Comparison of renal morphology in the Streptozotocin and the SHR/N-cp models of diabetes," Laboratory Investigation, vol. 84, no. 4, pp. 452464, 2004.

[34] M. P. O’Donnell, B. L. Kasiske, and W. F. Keane, "Glomerular hemodynamic and structural alterations in experimental diabetes mellitus," FASEB Journal, vol. 2, no. 8, pp. 2339-2347, 1988.

[35] H.-C. Huang and P. A. Preisig, "G1 kinases and transforming growth factor- $\beta$ signaling are associated with a growth pattern switch in diabetes-induced renal growth," Kidney International, vol. 58, no. 1, pp. 162-172, 2000.

[36] Z.-G. Xu, T.-H. Yoo, D.-R. Ryu et al., "Angiotensin II receptor blocker inhibits $\mathrm{p} 27^{\mathrm{Kip} 1}$ expression in glucose-stimulated podocytes and in diabetic glomeruli," Kidney International, vol. 67, no. 3, pp. 944-952, 2005.

[37] T. Pantsulaia, "Role of TGF-beta in pathogenesis of diabetic nephropathy," Georgian Medical News, no. 131, pp. 13-18, 2006.

[38] M. Romero, A. Ortega, A. Izquierdo, P. López-Luna, and R. J. Bosch, "Parathyroid hormone-related protein induces hypertrophy in podocytes via TGF- $\beta_{1}$ and $\mathrm{p} 27^{\mathrm{Kip} 1}$ : implications for diabetic nephropathy," Nephrology Dialysis Transplantation, vol. 25, no. 8, pp. 2447-2457, 2010.

[39] J. A. Ardura, S. Rayego-Mateos, D. Rámila, M. Ruiz-Ortega, and P. Esbrit, "Parathyroid hormone-related protein promotes epithelial-mesenchymal transition," Journal of the American Society of Nephrology, vol. 21, no. 2, pp. 237-248, 2010.

[40] J.-M. Fan, N. G. Yee-Yung, P. A. Hill et al., "Transforming growth factor- $\beta$ regulates tubular epithelial-myofibroblast transdifferentiation in vitro," Kidney International, vol. 56, no. 4, pp. 1455-1467, 1999.

[41] F. Strutz, M. Zeisberg, F. N. Ziyadeh et al., "Role of basic fibroblast growth factor-2 in epithelial-mesenchymal transformation," Kidney International, vol. 61, no. 5, pp. 1714-1728, 2002.

[42] W. Lieberthal and J. S. Levine, "Mechanisms of apoptosis and its potential role in renal tubular epithelial cell injury," American Journal of Physiology, vol. 271, no. 3, pp. R477-R488, 1996.

[43] J. A. Ardura, R. Berruguete, D. Rámila, M. V. Alvarez-Arroyo, and P. Esbrit, "Parathyroid hormone-related protein interacts with vascular endothelial growth factor to promote fibrogenesis in the obstructed mouse kidney," American Journal of Physiology, vol. 295, no. 2, pp. F415-F425, 2008.

[44] Y. Liu, "Epithelial to mesenchymal transition in renal fibrogenesis: pathologic significance, molecular mechanism, and therapeutic intervention," Journal of the American Society of Nephrology, vol. 15, no. 1, pp. 1-12, 2004. 
[45] R. Kalluri and E. G. Neilson, "Epithelial-mesenchymal transition and its implications for fibrosis," Journal of Clinical Investigation, vol. 112, no. 12, pp. 1776-1784, 2003.

[46] F. Strutz and G. A. Müller, "Renal fibrosis and the origin of the renal fibroblast," Nephrology Dialysis Transplantation, vol. 21, no. 12 , pp. 3368-3370, 2006.

[47] J. V. Bonventre, "Dedifferentiation and proliferation of surviving epithelial cells in acute renal failure," Journal of the American Society of Nephrology, vol. 14, no. 1, pp. S55-S61, 2003.

[48] B. Hinz, G. Celetta, J. J. Tomasek, G. Gabbiani, and C. Chaponnier, "Alpha-smooth muscle actin expression upregulates fibroblast contractile activity," Molecular Biology of the Cell, vol. 12, no. 9, pp. 2730-2741, 2001.

[49] S. Cheng and D. H. Lovett, "Gelatinase A (MMP-2) is necessary and sufficient for renal tubular cell epithelialmesenchymal transformation," American Journal of Pathology, vol. 162, no. 6, pp. 1937-1949, 2003.

[50] R. C. Harris and M. Martinez-Maldonado, "Angiotensin IImediated renal injury," Mineral and Electrolyte Metabolism, vol. 21, no. 4-5, pp. 328-335, 1995.

[51] J. Egido, "Vasoactive hormones and renal sclerosis," Kidney International, vol. 49, no. 2, pp. 578-597, 1996.

[52] A. Ortega, D. Rámila, A. Izquierdo et al., "Role of the reninangiotensin system on the parathyroid hormone-related protein overexpression induced by nephrotoxic acute renal failure in the rat," Journal of the American Society of Nephrology, vol. 16, no. 4, pp. 939-949, 2005.

[53] A. J. Allred, M. C. Chappell, C. M. Ferrario, and D. I. Diz, "Differential actions of renal ischemic injury on the intrarenal angiotensin system," American Journal of Physiology, vol. 279, no. 4, pp. F636-F645, 2000.

[54] J. Kontogiannis and K. D. Burns, "Role of AT1 angiotensin II receptors in renal ischemic injury," American Journal of Physiology, vol. 274, no. 1, pp. F79-F90, 1998.

[55] G. W. Long, D. C. Misra, R. Juleff, G. Blossom, P. F. Czako, and J. L. Glover, "Protective effects of enalaprilat against postischemic renal failure," Journal of Surgical Research, vol. 54, no. 3, pp. 254-257, 1993.

[56] R. C. Abdulkader, M. M. Yuki, A. C. M. Paiva, and M. Marcondes, "Prolonged inhibition of angiotensin II attenuates glycerol-induced acute renal failure," Brazilian Journal of Medical and Biological Research, vol. 21, no. 2, pp. 233-239, 1988.

[57] O. Lorenzo, M. Ruiz-Ortega, P. Esbrit et al., "Angiotensin II increases parathyroid hormone-related protein (PTHrP) and the type $1 \mathrm{PTH} / \mathrm{PTHrP}$ receptor in the kidney," Journal of the American Society of Nephrology, vol. 13, no. 6, pp. 1595-1607, 2002.

[58] C. J. Pirola, H.-M. Wang, A. Kamyar et al., "Angiotensin II regulates parathyroid hormone-related protein expression in cultured rat aortic smooth muscle cells through transcriptional and post- transcriptional mechanisms," The Journal of Biological Chemistry, vol. 268, no. 3, pp. 1987-1994, 1993.

[59] M. Nodat, T. Katoh, N. Takuwa, M. Kumada, K. Kurokawa, and Y. Takuwa, "Synergistic stimulation of parathyroid hormone-related peptide gene expression by mechanical stretch and angiotensin II in rat aortic smooth muscle cells," The Journal of Biological Chemistry, vol. 269, no. 27, pp. 17911-17917, 1994. 


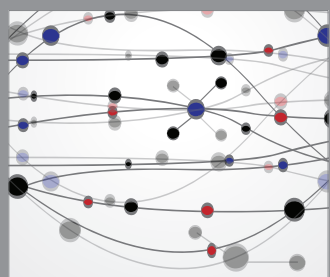

The Scientific World Journal
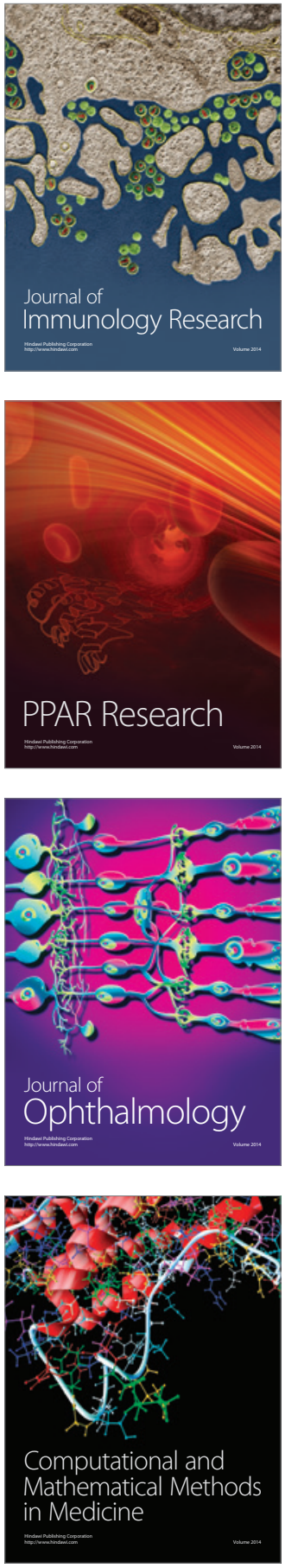

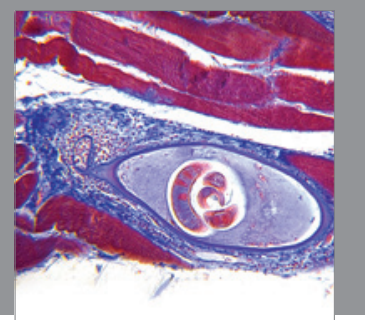

Gastroenterology

Research and Practice
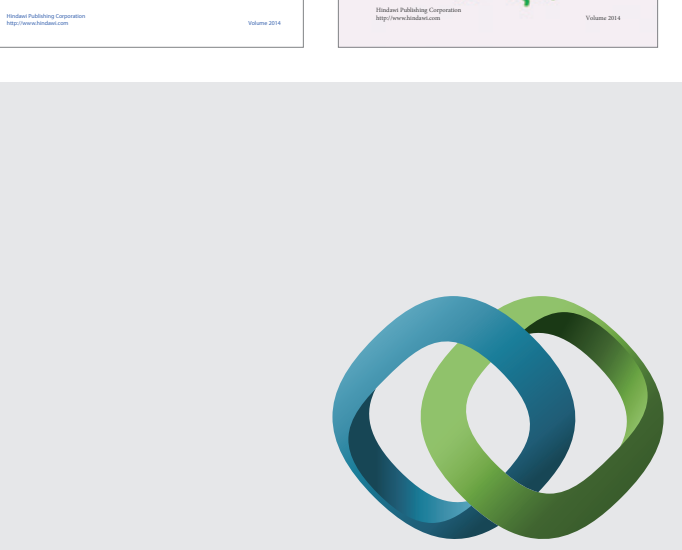

\section{Hindawi}

Submit your manuscripts at

http://www.hindawi.com
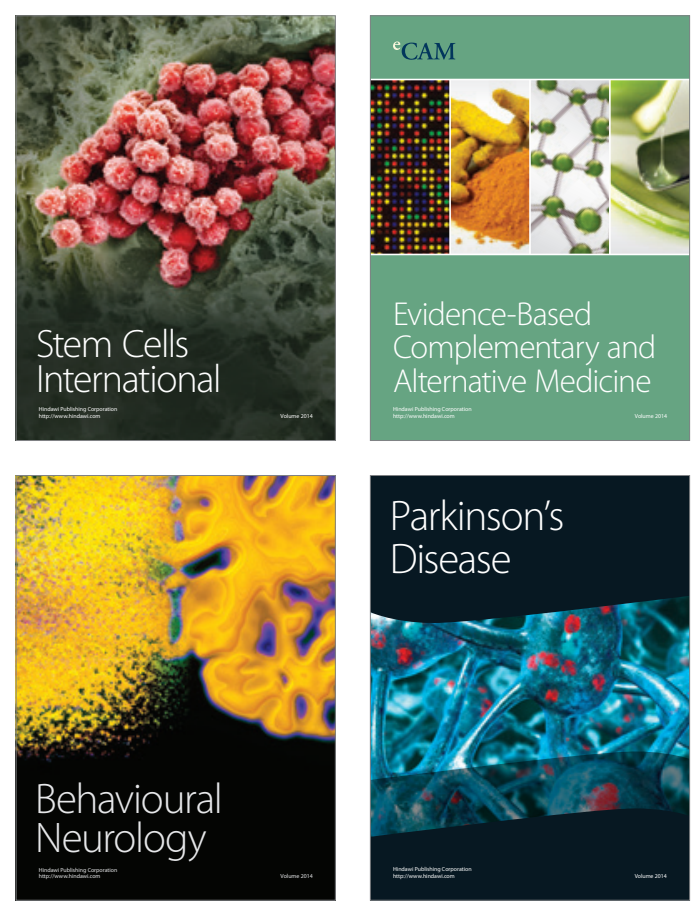

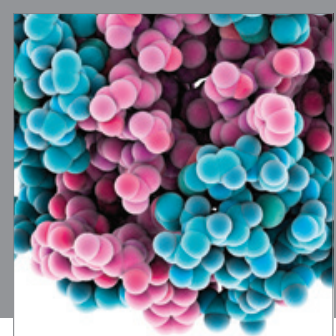

Journal of
Diabetes Research

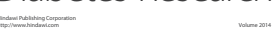

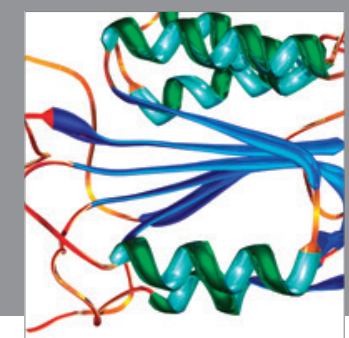

Disease Markers
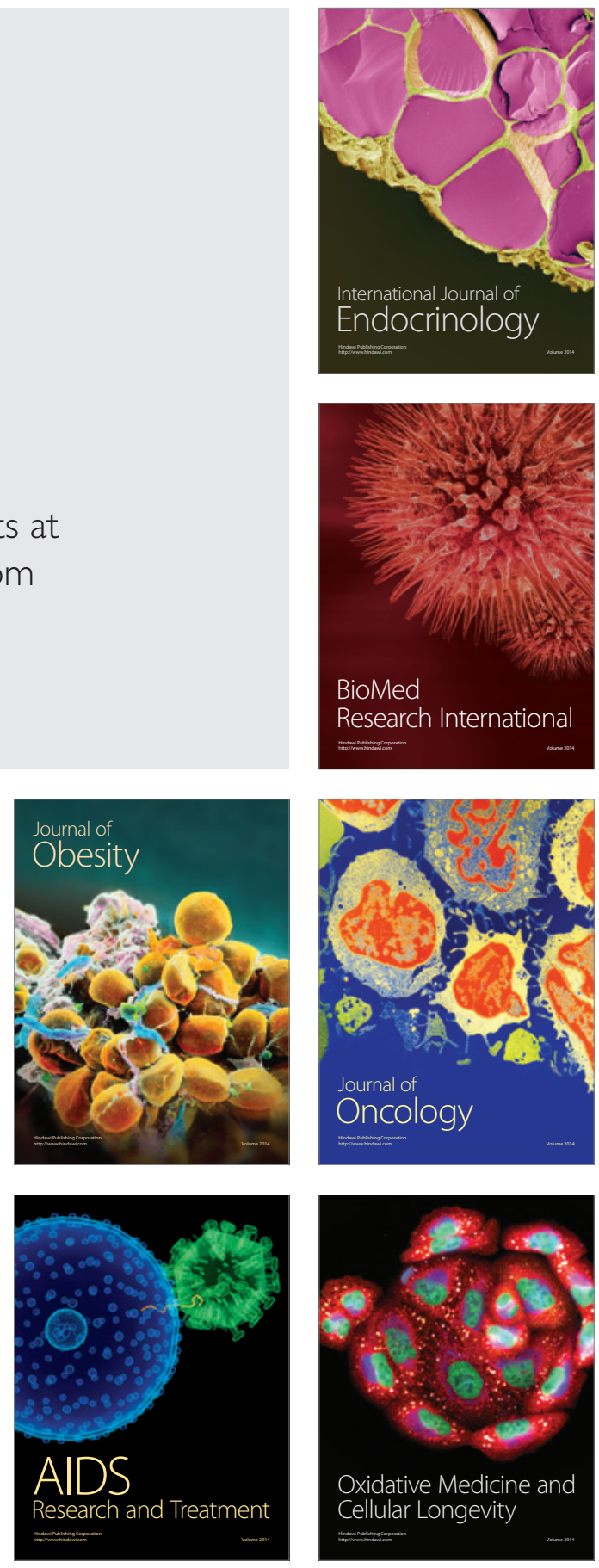